\title{
Design and Reliability of Clay Hardness Tester
}

\author{
Prapun AUNGATICHART, Sirithan JIEMSIRILERS and Shigetaka WADA \\ Department of Materials Science, Faculty of Science, Chulalongkorn University, Bangkok 10330 Thailand
}

\author{
粘土硬度計の設計と信頼性 \\ Prapun Aungatichart $\cdot$ Sirithan Jiemsirilers $\cdot$ 和田重孝 \\ チュラロンコン大学理学部材料科学科, 10330 バンコク, タイ
}

\begin{abstract}
The design and reliability of NGK type clay hardness tester, which has been used for measuring the hardness of plastic clay body in order to control and/or check the consistency of the ceramic clay dough in the ceramic production, were investigated. It was found that the hardness values were depended on the design of the hardness tester, and to a larger extent on the human error. The factors affecting the reliability of the hardness values were also discussed.

[Received July 19, 2006; Accepted August 24, 2006]
\end{abstract}

Key-words : Clay hardness tester, Reliability, Design

1. Introduction

Pfefferkorn ${ }^{1)}$ and Atterberg limit ${ }^{2)}$ are two major plasticity tests of soils and clays. Hardness tester with movable cone is also very popular in many ceramic industries because it is a portable, economical and convenient instrument for the determination of the hardness of clays. Moreover, the measurement can be performed quickly. It is said that Yamanaka type hardness tester was invented more than several ten years ago and more than 50,000 pieces have been sold and used in Japan. ${ }^{\left.3{ }^{3}, 4\right)}$ Yamanaka type and NGK type hardness testers are similar in their design and function. NGK type hardness tester was first imported into Thailand from Japan around 30 years ago, and it has widely spread in the country.

When we use NGK type tester as one of the standard evaluation methods for the property of clay, basic knowledge on the design, required standard and reliability of it is very important. Although there is some information on the usage of it, ${ }^{5)-7)}$ the NGK type tester comes in many designs according to producers and we can not find any basic data on the relation between the design and its performance. Therefore, it is interesting to perform an experiment to study the design and reliability of the hardness tester since the basic data obtained will benefit the reproducibility of the products. The ceramic body used in the experiment was local red clay for terracotta pottery in Thailand.

2. Design, structure and function of clay hardness tester

The appearance of NGK type hardness tester is shown in Fig. 1. It is composed of a cone, a spring, a hardness scale, a reading scale indicator, an adjustment screw, a spring stopper, a head cover and a case as schematically illustrated in Fig. 2. As demonstrated in Fig. 3, when the cone is pressed into the clay dough till reaching the supporting base, the reading scale indicator gradually moves from zero to larger number on the hardness scale and stops at some value depending on the hardness of the material. That is, the force resisting to the penetration of the cone into the dough balances against the force of spring. The hardness of the material is indicated by the hardness number engraved in the surface of the case. If the number is small, the dough is soft. On the contrary, if the number is large, the dough is stiff.

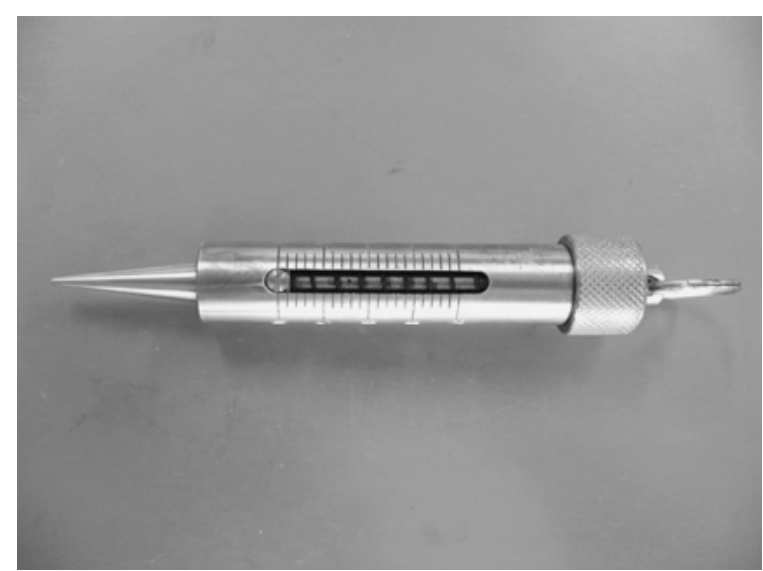

Fig. 1. Photograph of a hardness tester.

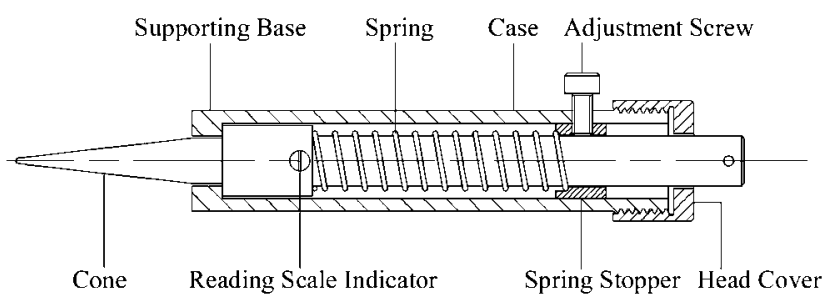

Fig. 2. Schematic structure of the hardness tester.

\section{Experiment}

3.1 Hardness testers used for the experiment

Five NGK type hardness testers having a little difference in the design were used for the experiment. All of the tools were two brand new (PN-01 and PN-02) and three of used (JOD01, POD-01 and POD-02) ones. The differences between the two groups of the tools were diameter, length and cone angle. The data of the diameter $(\phi)$ at the supporting base, the length $(l)$ and the angle $(\theta)$ of the cones are shown in Table 1. The hardness numbers engraved on the case were 0-25 for PN-01 and PN-02, and 0-20 for JOD-01, POD-01 


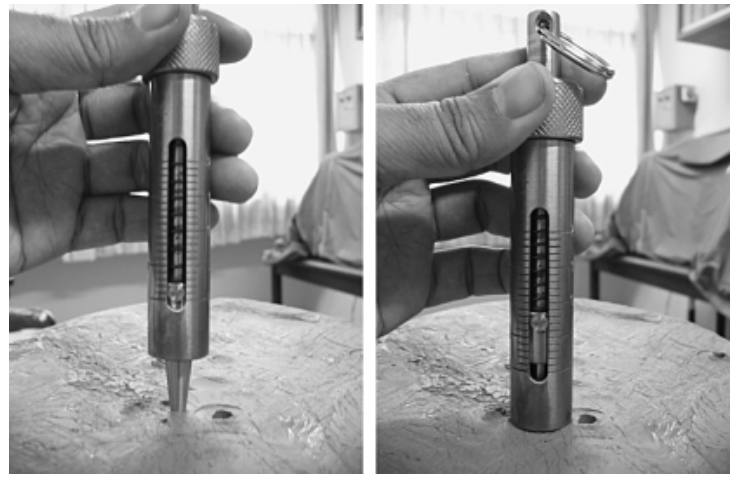

Fig. 3. Photographs illustrating the measurement method of the hardness tester.

Table 1. Diameter, Length and Angle of the Hardness Tester Cones

\begin{tabular}{|c|c|c|c|}
\hline Hardness tester & $\phi(\mathrm{mm})$ & $1(\mathrm{~mm})$ & $\theta=($ degree $)$ \\
\hline JOD-01 & 9.25 & 34.3 & 15 \\
\hline PN-01 & 9.75 & 44.7 & 12 \\
\hline PN-02 & 9.90 & 45.1 & 12 \\
\hline POD-01 & 9.55 & 33.7 & 16 \\
\hline POD-02 & 9.50 & 34.5 & 16 \\
\hline
\end{tabular}

and POD-02. One unit of the hardness scale is equal to $2 \mathrm{~mm}$. Thus, the length of hardness scale of $\mathrm{PN}-01$ and $\mathrm{PN}-02$ is 50 $\mathrm{mm}$, and $40 \mathrm{~mm}$ for JOD-01, POD-01 and POD-02.

The diameter and the length of the cone were measured by a vernier calliper. These values were difficult to measure accurately due to the cone figure. The angle of the cone tip $(\theta)$ was calculated from the diameter $(\phi)$ and the length $(l)$. From the values in Table 1, it was noticed that PN-01 and $\mathrm{PN}-02$ had longer and sharper cone figure than those of the rest. The spring constant of each tester was also measured. The values were $217.4 \mathrm{Nm}^{-1}$ for PN-01, PN-02, POD-01 and POD-02, and $202.1 \mathrm{Nm}^{-1}$ for JOD-01.

\subsection{Sample preparation}

The plastic red clay (Ratchaburi, Thailand) with some amount of sand and grog was used for the hardness measurement. The test pieces were extruded to cylindrical shape with dimension approximately $20 \mathrm{~cm}$ in both diameter and length. Afterwards these specimens were wrapped with plastic film and kept for one night to homogenize the moisture.

\subsection{Experimental method}

To study the effect of human error on the measurement, five persons joined the experiment. Each person was denoted as person A, B, C, D and E. The conditions of the measurement were as follows:

(a) Before training and calibration of the testers

Five hardness testers were used at the condition as received. Each person used 5 hardness testers alternately without any instruction. Ten measurements were done for each person/ tool pair.

(b) After training without calibration of the testers

The same testers were used. The training instructions were as follows: (i) the hardness tester should be pressed perpendicular to the surface of the test piece and (ii) it should be pressed slowly and then held until the drift of the reading scale stops. The hardness number should be read after the drift stops.

(c) After training and calibration of the testers

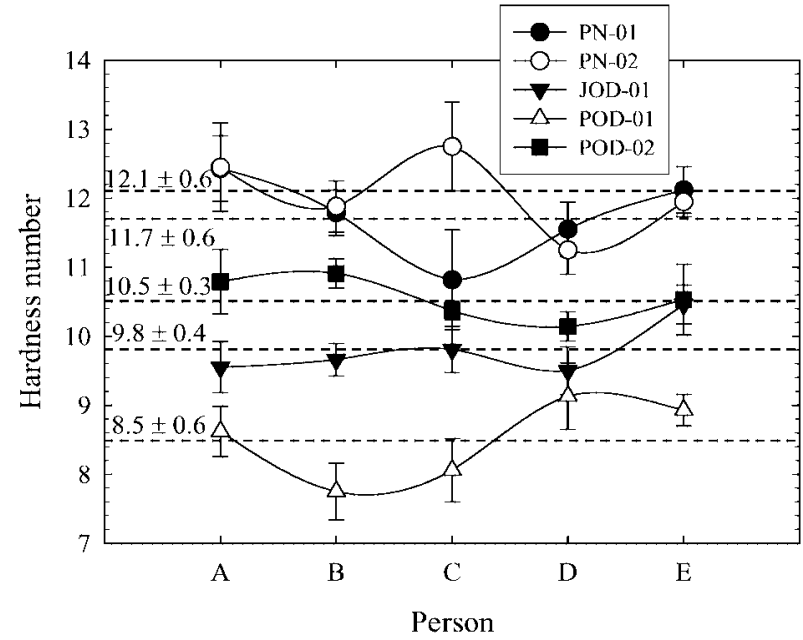

Fig. 4. Hardness numbers obtained by 5 persons before training using non calibrated tools.

The persons who used the hardness testers were trained and the hardness testers were calibrated as follows:

i) At a fixed load

All hardness testers were calibrated by adjusting the force of the spring through the adjustment screw. The spring was so calibrated that the hardness number showed 10 when $7.8 \mathrm{~N}$ of the load was applied to it.

ii) At various loads

To know the effect of the spring strength on the deviation of the hardness number, only one tester (JOD-01) was selected for the experiment, because we thought that the tendency of the other testers might be similar to JOD-01. The spring of the selected tester was set at soft, medium and hard conditions by adjusting the spring length using adjustment screw. At these three conditions, the hardness number of 5 corresponded to the loads of $3.7,6.8$ and $8.8 \mathrm{~N}$, respectively. The spring lengths were $73.3 \mathrm{~mm}$ for soft, $60.7 \mathrm{~mm}$ for medium and 53.9 $\mathrm{mm}$ for hard conditions. Three persons measured the hardness at these conditions.

\section{Results and discussion}

4.1 Hardness before training and calibration of the testers

The hardness numbers obtained by 5 persons before training and calibration are shown in Fig. 4. The average hardness numbers with standard deviations due to personnel obtained for each tool are $8.5 \pm 0.6$ for POD-01, $9.8 \pm 0.4$ for JOD-01, $10.5 \pm 0.3$ for POD-02, $11.7 \pm 0.6$ for $\mathrm{PN}-01$ and $12.1 \pm 0.6$ for PN-02. In comparison, these standard deviations are less reliable due to calculation from such a few numbers of data (only 5). The error bars shown in the figure are the standard deviation for each person/tool pair which is calculated using ten numbers of data. Hence, they are more reliable. Considering all the data from 5 persons for each tool, the hardness number values wildly fluctuated. The causes of those fluctuations are thought to be the sum of the human error, difference among non calibrated tools, intrinsic accuracy limit coming from the tool, and the hardness inhomogeneity of the dough.

During the experiment, it was found that the speed of pressing was different from person to person. When the cone was pressed quickly, reading scale showed large number, and after pressing the hardness number tended to drift down with time. When the cone was pressed slowly, there was no overrun in the 


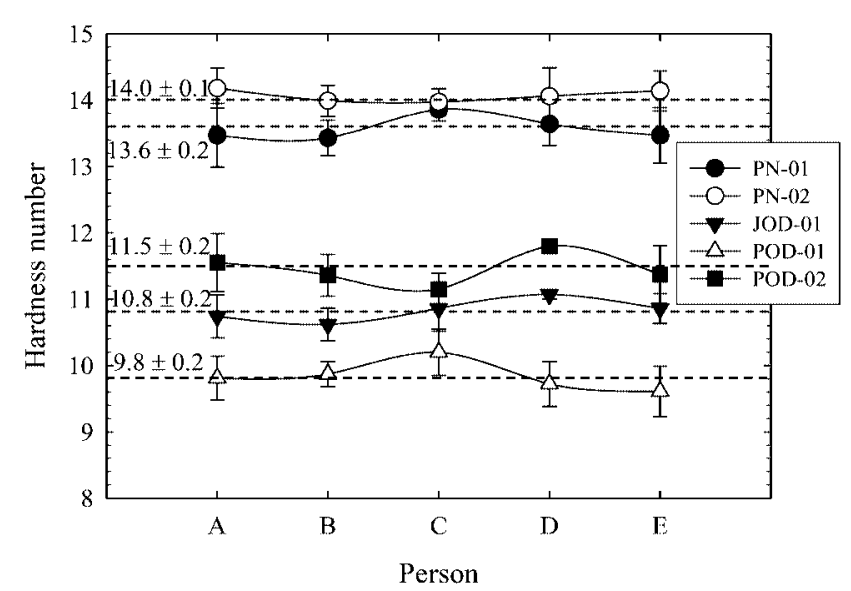

Fig. 5. Hardness numbers obtained by 5 persons after training using non calibrated tools.

hardness number. When the cone was pressed slantingly to the surface of the dough, the hardness number was a little smaller than the value when it was pressed vertically.

Considering the observations mentioned above, it is supposed that the human errors are largely caused by lacking of standard testing procedure, i.e. the different speed of pressing the cone, reading time of the hardness number after pressing and pressing angle of the cone to the dough surface.

4.2 Hardness after training without calibration of the testers

Considering the results of 4.1 presented in Fig. 4, the same five persons were trained that the tool should be pressed perpendicular to the surface of the dough, the pressing speed should not be too quick, and the hardness number should be read after the drift of the reading scale almost stopped. Before the formal experiment, they trained themselves following the instructions.

The hardness numbers obtained by 5 persons after training without calibration of tools are shown in Fig. 5. The average hardness numbers with standard deviations obtained from the 5 tools are $9.8 \pm 0.2$ for POD-01, $10.8 \pm 0.2$ for JOD-01, 11.5 \pm 0.2 for POD-02, $13.6 \pm 0.2$ for $\mathrm{PN}-01$ and $14.0 \pm 0.1$ for $\mathrm{PN}-02$. After training, the results clearly indicate that the fluctuations of the data of each hardness tester are less than those of before training. This means that the testing procedure is one of the major causes of the fluctuation of the data. However, the hardness number of each hardness tester still varies because the hardness testers have not been calibrated.

Incidentally, the average hardness numbers shown in Figs. 4 and 5 are not significantly different. Comparing the hardness value of each tool, the differences are 1.3 for POD-01, 1.0 for POD-02, 1.0 for JOD-01, and 1.9 for PN-01 and PN-02. The difference may also come from the variation of the dough. However, it can be concluded that the absolute value was different for the tools of different design. We could not make clear the cause of the difference in this experiment.

4.3 Hardness after training and calibration of the testers

(1) At a fixed load

The relationship between hardness number and the person after training using the calibrated tools is shown in Fig. 6. The graph shows that the data are obviously separated into two groups. Group 1 consisting of POD-01, POD-02 and JOD-01 shows lower hardness values on the graph. The average hardness numbers are $9.4 \pm 0.2$ for POD-01, 9.7 \pm 0.3 for POD-02 and $9.7 \pm 0.1$ for JOD-01. It is noted that the hardness values

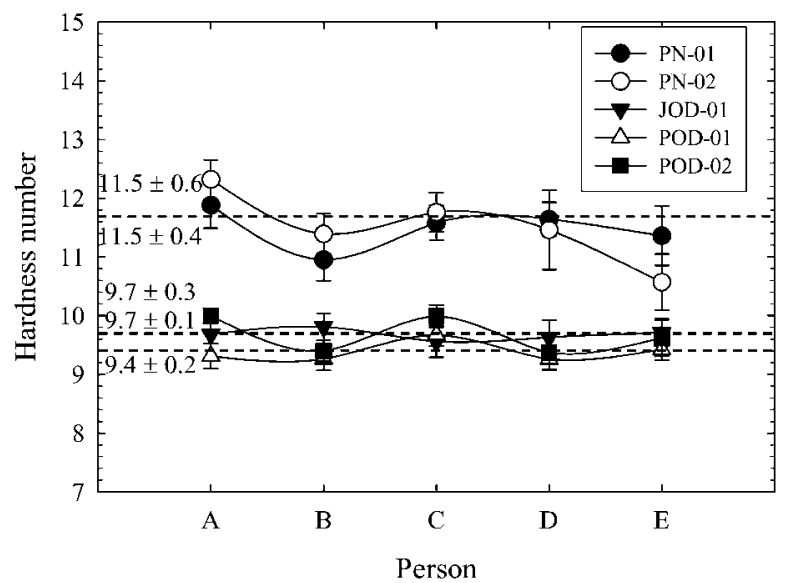

Fig. 6. Hardness numbers obtained by 5 persons after training using calibrated tools.

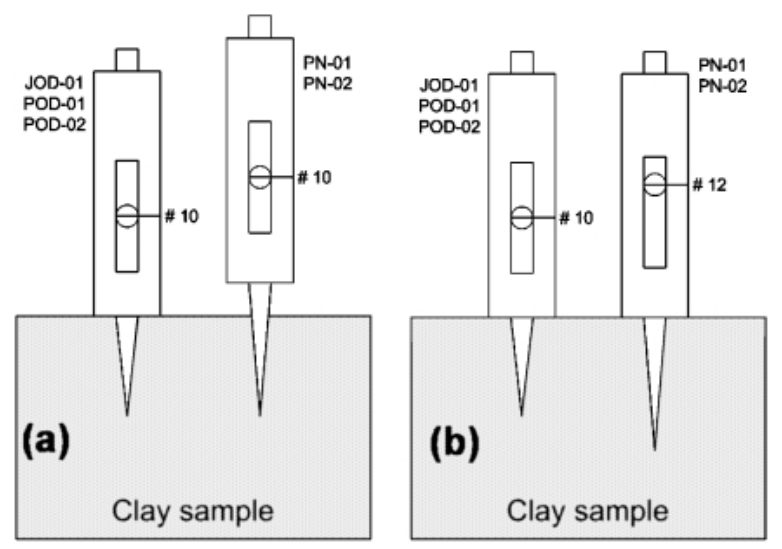

Fig. 7. Schematic structures illustrating the measured results after calibration and training using the hardness testers with different designs.

are insignificantly different when the tools were calibrated. The difference of the decimal numbers (about 0.3) is regarded as the error from reading. $\mathrm{PN}-01$ and $\mathrm{PN}-02$, which indicated higher hardness values, belong to group 2. Their hardness values are the same value of 11.5. This is due to the distinction between the designs of the two tool groups, especially the length of their cones as mentioned in 3.1. It implies that even though the hardness testers were calibrated so that the hardness number shows 10 at the same load of $7.8 \mathrm{~N}$, still the hardness number values corresponding to the cone area resisting to the force of spring is dependent on the design. This phenomenon can be schematically illustrated as Fig. 7. Figure 7(a) depicts that the supporting base of $\mathrm{PN}-01$ and $\mathrm{PN}-02$ can not reach the clay surface while the hardness number on the scale become 10. In the case of JOD-01, POD-01 and POD-02, which have the shorter cones, their supporting bases reach the clay surface. This condition is instructed as the corrective condition for measuring the hardness of the clay. If $\mathrm{PN}-01$ and $\mathrm{PN}-02$ are used in the corrective way, the additional force has to be applied to them to push their supporting bases to reach the surface of the samples as shown in Fig. 7(b). As a result, the hardness number becomes 12 instead of 10 (see Fig. 7 (b) ). Hence, this is the reason why the hardness number from PN-01 and PN-02 are always higher than those of JOD-01, 


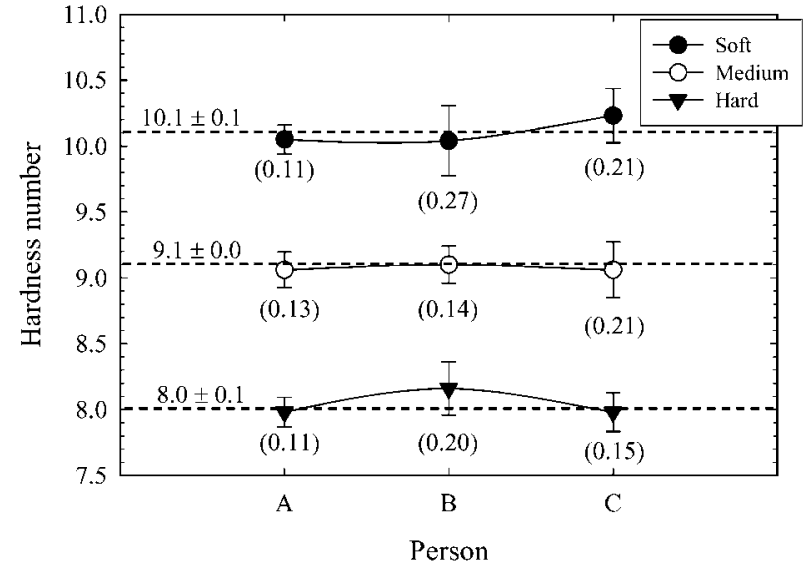

Fig. 8. Hardness number obtained by 3 persons at different calibration (numbers in the parenthesis show the standard deviation for each person/tool measurement).

POD-01 and POD-02. It is concluded that the hardness values obtained from the testers of the same type but of different designs as described above can not be compared. It means that the hardness number values can be compared only when the design, especially the figure of the cone is exactly the same.

The large deviations of the average hardness numbers of PN-01 and PN-02 come from the larger value obtained by person $\mathrm{A}$ and the small value obtained by person $\mathrm{E}$. It is possible that they might not follow the measurement instructions.

\section{(2) At various loads}

When spring is calibrated "Hard" the cone sticks into the clay specimen deeply and when spring is calibrated "Soft", it is in reverse. When the cone does not stick in deeply, a small area of cone resists the force of the spring. In such a condition, we think that the deviation of the measured hardness value may increase due to a small cone area.

To know the effect of the spring strength on the hardness values and deviations of the hardness testers, the springs was set at soft, medium and hard conditions as described in 3.3 (c(ii)). The measured values of three persons are plotted in Fig. 8. The average hardness values from three persons are about 10 at soft, 9 at medium and 8 at hard. The deviation of the hardness number is not significantly different as shown in the error bar and number in parenthesis in Fig. 8. As a result, it is suggested that the calibration load of the spring is not restricted to some values. One of the calibration requirements is that the reading scale indicator of the tool should be set to the center of the hardness number in the case.

\section{Conclusions}

The factors affecting the hardness values of the clay sample measured with the different design hardness testers can be drawn as follows:

(1) The human error is one of the big factors affecting the deviation of the hardness number value and when a person is guided properly, the human error can be decreased easily.

(2) The design of the hardness tester, especially the figure of the cone, strongly affects the hardness number. When the figure of the cone is different, the testers can not be calibrated to give the similar hardness value even the spring is set at the same load. The hardness number values can be compared only when the testers have the same design.

(3) The hardness number values obtained are changed by the calibration. The hardness number values of each tester give a narrow distribution after the calibration.

Acknowledgements The authors wish to thank Mr. Takeshi Yoshikawa and Assoc. Prof. Dr. Supatra Jinawath for their comments and suggestions.

\section{References}

1) Dietzel, V. A., Ber. Dtsch. Keram. Ges., Vol. 45, pp. 63-66 (1968) [in German].

2) ASTM D 4318-05, 2005, Standard test methods for liquid limit, plastic limit, and plasticity index of soils. Annual book of ASTM standards, Philadelphia.

3) Yamanaka, K. and Matsuo, K., Dojyo Hiryo Gakkaishi (J. of Japanese Society of Soil Science and Plant Nutrition), Vol. 33, pp. 343-347 (1962) [in Japanese].

4) http://www.fujiwara-sc.co.jp/catalog/yamanaka.html (in Japanese).

5) Katoh, N. et al., US Patent 5573 982, 12 November 1996.

6) Kawasaki, S. et al., US Patent 5866 275, 2 February 1999.

7) Kawasaki, S. et al., US Patent 6179 884, 30 January 2001. 